\title{
Direct measurement of salt-mineral repulsion using atomic force microscopy
}

\author{
Andrea Hamilton,* Vasileios Koutsos and Christopher Hall \\ Received 31st July 2009, Accepted 28th April 2010 \\ First published as an Advance Article on the web 22nd June 2010 \\ DOI: $10.1039 / b 915709 c$
}

The disjoining pressure between a mineral and soluble salt crystal in concentrated aqueous solution has been successfully measured with atomic force microscopy.

Salt crystals can grow in the pores of stone, generating stress in the host matrix and leading to serious damage ${ }^{1}$ in building materials and works of art. Crystallization stress is also a cause of natural rock weathering in terrestrial and planetary landform alteration. ${ }^{2}$ The ability of salt crystals to cause damage depends on the existence of a film of solution between the crystal and pore wall to allow continued growth, and a repulsive interaction between the crystal and the pore to maintain the film. ${ }^{3}$ If the salt-mineral interaction through the film of solution is attractive, the salt crystal and pore wall make adhesive contact and no damage should be observed. The thickness of the solution film $^{3}$ is estimated to be a few $\mathrm{nm}$, highlighting the importance of understanding and eventually tailoring the nanoscale interaction between salt crystals and rock forming minerals. The disjoining pressure is the force needed to push unit area of crystal and of pore wall into hard contact, and therefore is the upper bound on the real crystallization pressure. Our aim was to measure directly for the first time the disjoining pressure between a rock-forming mineral (using silica as an analogue for quartz) and a soluble salt crystal. Sodium sulfate, the most damaging ${ }^{2}$ salt, has such complex hydrate chemistry ${ }^{4}$ that force-distance AFM work was impractical. Therefore we used potassium sulfate (arcanite), chosen for its high solubility and its stability in air.

Potassium sulfate crystals were grown by evaporation on a freshly cleaved mica sheet to produce a smooth surface. Crystals were attached to the AFM stub with Araldite ${ }^{\mathbb{R}}$ 2014 and left to cure at room temperature in a desiccator for several weeks. We used a Multimode/Nanoscope IIIa (Veeco) AFM with a fluid cell. Room temperature was maintained at $21{ }^{\circ} \mathrm{C}$. Heating from laser irradiation raises the temperature in the fluid cell by $3-4{ }^{\circ} \mathrm{C}$. ${ }^{5}$ Better surface stability was achieved by injecting solutions saturated at ca. $8{ }^{\circ} \mathrm{C}$ above room temperature with added crystal growth inhibitor. Potassium sulfate solutions saturated at $29^{\circ} \mathrm{C}$ were used $(0.595 \mathrm{M}$, Fisher AR grade, $+99 \%$ ), to which 5 ppm of $\mathrm{Cr}^{3+}$ was added to the solution as $\mathrm{KCr}\left(\mathrm{SO}_{4}\right)_{2} \cdot 12 \mathrm{H}_{2} \mathrm{O}$ (Sigma-Aldrich, ACS reagent grade, $+99 \%)$.

Solutions were injected through syringe filters (pore size $0.2 \mu \mathrm{m})$ to remove foreign particles. Custom-made $\mathrm{CrAu}$

School of Engineering and Centre for Materials Science \& Engineering, The University of Edinburgh, The King's Buildings, Edinburgh,UK.E-mail: andrea.hamilton@ed.ac.uk;

Fax: + 44 (0)131-650-6554; Tel: + 44 (0)131-650-5693
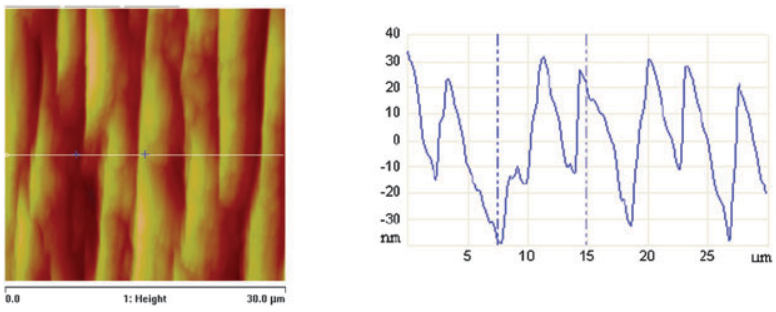

Fig. $130 \times 30 \mu \mathrm{m}$ scan of a templated arcanite surface in $0.595 \mathrm{M}$ solution with no inhibitor. A section through the image is shown (left). Peak-to-trough max and RMS roughness are $115 \mathrm{~nm}$ and $20.9 \mathrm{~nm}$ respectively.
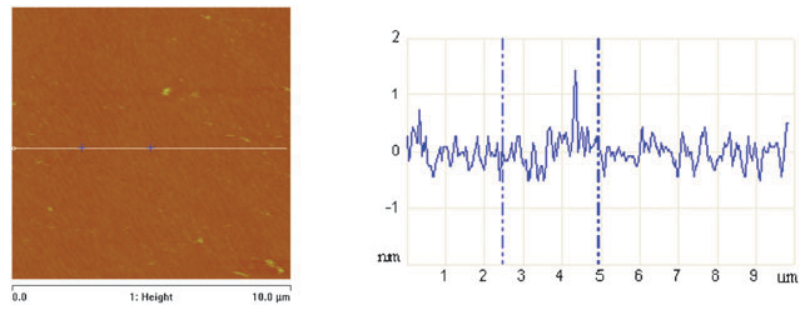

Fig. $210 \times 10 \mu \mathrm{m}$ scan of a templated arcanite surface in $0.595 \mathrm{M}$ solution with $5 \mathrm{ppm} \mathrm{Cr}^{3+}$. A section through the image is shown (left). Peak-to-trough max and RMS roughness are $9.18 \mathrm{~nm}$ and $0.31 \mathrm{~nm}$ respectively. 
water under an optical microscope, and others imaged and mapped by SEM with EDX facility. Neither of the two crystals used for the data presented here contained any detectable trace of mica.

After imaging the surface, $4 \mathrm{~mL}$ of solution was flushed through the cell and 155 force curves were taken using the silica colloid cantilever at a piezo speed of $0.1 \mathrm{~Hz}$. The raw tip deflection vs. piezo movement data were converted to forceseparation curves using the measured spring constant and deflection sensitivity measured from the top part of the approach curve. The point of zero distance was determined as described by Butt et al. ${ }^{6}$ by extrapolating between the contact region of the curve and the non-contact region at large separations. The resulting force-separation curves were averaged to produce a single curve which was smoothed using a 5-point FFT routine. The Derjaguin approximation is used to obtain the corresponding interaction free energy per unit area between two flat plates and the resulting curve differentiated to achieve the disjoining pressure $v s$. separation (Fig. 3).

It is clear that no obvious minima, primary or secondary, are observed and that the interaction is essentially repulsive at all separations. Modeling the system using the algorithm of $\mathrm{Chan}^{7}$ which solves numerically the non-linear PoissonBoltzmann equation for asymmetrical electrolytes but modified to include dissimilar surfaces and van der Waals interactions predicts an attraction beginning at $0.5-1.5 \mathrm{~nm}$ (see Fig. 3). Modification for dissimilar surfaces was achieved by integrating twice for $\kappa L$ (inverse Debye length multiplied by surface separation $L$ ), with the lower limit of integration being the potential minimum in both cases, but the upper limits set at the surface potential for the two dissimilar surfaces. The total $\kappa L$ value is the sum of the two $\kappa L$ values which together equal the dimensionless distance between the plates. van der Waals forces are calculated for a sphere and a plane and converted to pressure via the Derjaguin approximation and differentiating the equation for interaction free energy per unit area. ${ }^{8}$ The Hamaker constant used is calculated for arcanite/silica-potassium sulfate solution using the Lifshitz approximation; retardation effects are not considered. Modeling was carried out for constant potential and constant charge, as shown in Fig. 3, which are generally considered the standard lower and upper boundaries, respectively, in DLVO theory. What is clear from the fit is that DLVO does not adequately describe either the length scale or the nature of the interaction at short distances but that constant charge (CC) is a slightly better fit than constant potential (CP). Bostrom et al. ${ }^{9}$ state that at

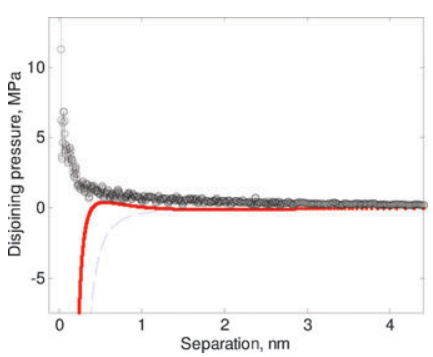

Fig. 3 Disjoining pressure calculated from smoothed average of 155 force curves (black circles). DLVO CP fit (dashed line) and CC fit (dotted line). concentrations above $0.1 \mathrm{M}$ DLVO theory is flawed and describe a method for taking the image and dispersion forces between ions into account. The result is a stronger attraction than that predicted by DLVO (constant potential) which does not describe the results presented here. An approximation to the shape of the curve, up to gap length of $c a .0 .5 \mathrm{~nm}$, can be obtained with the constant charge boundary condition at surface potentials of $-22 \mathrm{mV}$ and $-19 \mathrm{mV}$ for silica and arcanite (Fig. 3). Reducing the input surface potential to much lower values in both the $\mathrm{CC}$ and $\mathrm{CP}$ models produces a primary minimum with little or no repulsion visible at any separation. This is not surprising since at high salt concentrations the charge and potential generally tend towards zero and the electrostatic part of DLVO is screened out leaving the $\mathrm{vdW}$ contribution only. In salt crystallization damage in rocks, crystal growth must occur in concentrated supersaturated solution with little or no electrostatic component. This suggests the repulsive regime observed here applies to other salt-mineral systems for which crystallization damage is observed.

Very little literature exists on surface interactions in concentrated $(>0.1 \mathrm{M})$ solution and even less on dissimilar surfaces. Long range hydration repulsion, extending out to $7 \mathrm{~nm}$, between mica sheets at high $\mathrm{NaCl}$ concentration (60-600 mM) was observed by Alcantar et al. ${ }^{10}$ who remarked that hydrated $\mathrm{Na}^{+}$could push apart mica sheets forced together under $10-50 \mathrm{MPa}$ of pressure by up to $3 \mathrm{~nm}$. They attribute this long range interaction to hydrated $\mathrm{Na}^{+}$binding to negative sites on the mica surface. Work on silica ${ }^{11,12}$ shows that less hydrated ions such as $\mathrm{K}^{+}$and $\mathrm{Cs}^{+}$bind more easily to the silica surface and that at high concentrations $(\sim 1 \mathrm{M})$ $\mathrm{K}^{+}$ions condensed on the surface can produce a surface charge inversion. In the case of charge inversion, they describe an extra short-range repulsion that takes place at separations $<2 \mathrm{~nm}(0.5 \mathrm{M} \mathrm{CsCl})$ after a pronounced $\mathrm{vdW}$ attraction and describe it as a short range electrical double layer repulsion. Ion and surface specific effects and long range non-DLVO forces are also evident in the force-distance experiments of Veeramasuneni et al. ${ }^{13}$ using a silica colloid tip and $\mathrm{NaCl}$ and $\mathrm{KCl}$ crystals in saturated solution. With $\mathrm{NaCl}$ they find a strong attraction extending to $\sim 17 \mathrm{~nm}$ and with $\mathrm{KCl}$ a purely repulsive interaction to $\sim 4 \mathrm{~nm}$. Although they do not calculate disjoining pressure for either system their results suggest $\mathrm{KCl}$ has the potential to be damaging to siliceous stones where $\mathrm{NaCl}$ is not. Ion and crystal face specific interactions are described by Finot et al., ${ }^{14}$ who measured forces between silica and gypsum and pairs of gypsum crystals in solutions an order of magnitude less concentrated than the solutions used here.

Our results show a long range repulsion, consistent with that of Alcantar et al. , $^{10}$ and no strong attraction. A very slight secondary minimum (not shown) is found at $c a .13 \mathrm{~nm}$ with a force value of $32 \mathrm{pN}$ followed by a substantial repulsion and no observable primary minimum. $\zeta$-Potential measurements were made (Malvern, Zetasizer Nanoseries) on particles of arcanite (not more than $10 \mu \mathrm{m}$ in diameter) and silica (Aston Chemicals UK, $3 \mu \mathrm{m}$ diameter, $50 \AA$ pore size) in $0.595 \mathrm{M}$ $\mathrm{K}_{2} \mathrm{SO}_{4}$ with $5 \mathrm{ppm} \mathrm{Cr}^{3+}$ which suggested a near zero but slightly negative $\zeta$-potential for both particles. As the diffuse 
double layer shrinks, the $\zeta$-potential tends towards zero but the standard deviation, which is $\pm 3-4 \mathrm{mV}$ means that the absolute value is a rough guide. The high solubility of arcanite crystals makes it impossible to measure the $\zeta$-potential in dilute solution, which would be more consistent with standard

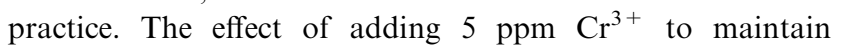
arcanite surface stability was investigated through $\zeta$-potential measurements on silica spheres in 0.6 and $6 \mathrm{mM} \mathrm{K}_{2} \mathrm{SO}_{4}$ solutions with and without $5 \mathrm{ppm} \mathrm{Cr}^{3+}$. Results were $-25.6 \mathrm{mV}\left(0.6 \mathrm{mM}\right.$, no $\left.\mathrm{Cr}^{3+}\right),-26.5(0.6 \mathrm{mM}, 5 \mathrm{ppm}$ $\left.\mathrm{Cr}^{3+}\right),-7.0 \mathrm{mV}\left(6 \mathrm{mM}\right.$, no $\left.\mathrm{Cr}^{3+}\right)$ and $-7.3 \mathrm{mV}(6 \mathrm{mM}$, $5 \mathrm{ppm} \mathrm{Cr}^{3+}$ ) which are consistent with the fact that the Debye

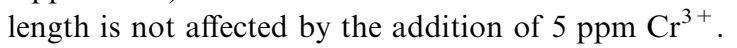

When the experimental curve in Fig. 3 is fitted to a second order exponential, we obtain values of 2.3-2.7 MPa for the disjoining pressure at $0.165-0.2 \mathrm{~nm} .{ }^{10}$ The decay length of the repulsion is $2.9 \mathrm{~nm}$, an order of magnitude higher than the Debye length but fairly consistent with the non-DLVO repulsion observed by Alcantar et al., ${ }^{10}$ who report a decay length of $1 \mathrm{~nm}$. Beyond the repulsion, hard contact is reached. Although a primary minimum is not observed, an adhesion force is measured on retraction. Calculating ${ }^{15}$ the pull-off force from measured surface roughness and the estimated van der Waals force gives a value about 25 times greater than the measured pull-off force. This suggests that either the surface roughness is much higher in the area sampled by the colloid tip or that even at hard contact, repulsive non-DLVO forces effectively reduce the tip-sample adhesion.

An ultimate aim of studying the disjoining pressure is to find a method of tailoring the interaction at small separations to promote an attractive interaction and reduce damage from salt crystallization. Zohar et al. ${ }^{16}$ find charge inversion between silica surfaces in the presence of $Z=3$ ions which dramatically alters their force curves from a long range repulsion to a much shorter range attraction. To see if this can occur in silica/ arcanite, saturated potassium sulfate solutions were prepared at $29{ }^{\circ} \mathrm{C}$ with 5,50 and $500 \mathrm{ppm}$ of crystal growth inhibitor. An equilibration time of $15 \mathrm{~min}$ was allowed before force curves were captured. At least $4 \mathrm{~mL}$ of each new solution was flushed through the cell prior to data collection. 155, 47 and 50 force curves were analyzed for the 5, 50 and $500 \mathrm{ppm}$ solutions respectively.

Fig. 4 shows a typical set of force-separation curves with additions of 5, 50 and $500 \mathrm{ppm}$ inhibitor $\mathrm{Cr}^{3+}$. It is clear that

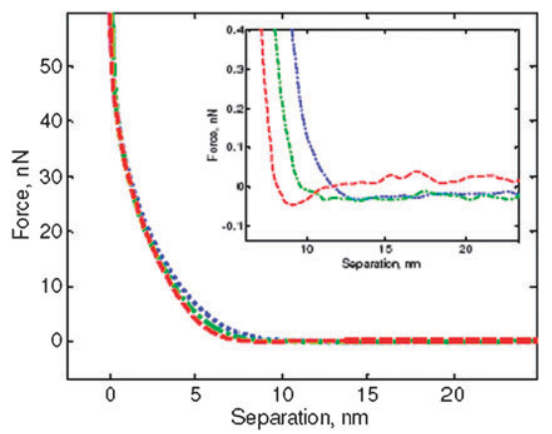

Fig. 4 Force-separation curves for the three solutions of $0.595 \mathrm{M}$ potassium sulfate containing $5 \mathrm{ppm}$ (dotted line), $50 \mathrm{ppm}$ (dot-dash line) and $500 \mathrm{ppm}$ (dashed line) of $\mathrm{Cr}^{3+}$. at $500 \mathrm{ppm}$, an attraction appears on approach at $c a .9 \mathrm{~nm}$ but that it is still followed by repulsion. The Debye length changes very little on addition of $500 \mathrm{ppm} \mathrm{Cr}^{3+}$ but $\zeta$-potential measurements suggest that surface charge inversion by $500 \mathrm{ppm}$ of $\mathrm{Cr}^{3+}$ is more effective than predicted charge inversion from very high concentrations of monovalent cations, ${ }^{16} 1.19 \mathrm{M} \mathrm{K}^{+}$ in this case. $\zeta$-Potential values for silica spheres in $0.6 \mathrm{mM}$ $\mathrm{K}_{2} \mathrm{SO}_{4}$ with 5,50 and $500 \mathrm{ppm}$ added are $-26.5,-24.2$ and $-19.2 \mathrm{mV}$ respectively. For $6 \mathrm{mM} \mathrm{K}_{2} \mathrm{SO}_{4}$ with 5,50 and $500 \mathrm{ppm} \mathrm{Cr}^{3+}$ added the $\zeta$-potential results are $-7.3,-4.7$ and $-1.4 \mathrm{mV}$ respectively. At $500 \mathrm{ppm} \mathrm{Cr}^{3+} 6$ out of the 11 samples analyzed had positive values under $+3 \mathrm{mV}$ but again a standard deviation of $\pm 3-4 \mathrm{mV}$ per measurement. While the absolute value of samples at close to $0 \mathrm{mV}$ cannot be taken too literally, the trend towards charge inversion is clear. Analysis of arcanite crystals in $0.595 \mathrm{M} \mathrm{K}_{2} \mathrm{SO}_{4}$ solutions with the same set of $\mathrm{Cr}^{3+}$ additions also suggests the surface could be positively charged in $500 \mathrm{ppm} \mathrm{Cr}^{3+}$. Ion correlation effects (vdW forces between polarisable counterions) are responsible for the attraction at $c a .9 \mathrm{~nm}$ and the repulsion at shorter range suggests $\mathrm{Cr}^{3+}$ is not tightly bound to the surface. The use of $\mathrm{Cr}^{3+}$ shows that it is possible to tailor salt-mineral interactions, for example through charge inversion. This opens a possible important new approach to prevent salt crystallization damage in building materials.

Results from a total of 455 measurements on two crystals at $\mathrm{Cr}^{3+}$ concentrations of 5,50 and $500 \mathrm{ppm}$ were analysed to arrive at a disjoining pressure for silica-arcanite in potassium sulfate solution of 2.4 to $4 \mathrm{MPa}$. This is at the lower end of the range (ca. 2-9 MPa) of tensile strength for most sedimentary rocks ${ }^{17}$ and may explain damage through slow crack propagation in cyclical crystallization tests. Charge inversion by $\mathrm{Cr}^{3+}$ produces an attraction at long range between silica and arcanite with repulsion at short range. This is the first measurement of disjoining pressure and charge inversion in a salt-mineral system and proof that a repulsive interaction can exist between minerals and salt crystals in salt solution.

\section{Notes and references}

1 N. Tsui, R. J. Flatt and G. W. Scherer, J. Cult. Heritage, 2003, 4, 109.

2 A. S. Goudie and H. A. Viles, Salt Weathering Hazards, Wiley, Chichester, 2007.

3 G. W. Scherer, Cem. Concr. Res., 1999, 29, 1347.

4 A. Hamilton and C. Hall, J. Anal. At. Spectrom., 2008, 23, 840.

5 A. Mauri and M. J. Moret, J. Cryst. Growth, 2000, 208, 599.

6 H.-J. Butt, B. Cappella and M. Kappl, Surf. Sci. Rep., 2005, 59, 1.

7 D. Y. C. Chan, J. Colloid Interface Sci., 2002, 245, 307.

8 J. N. Israelachvili, Intermolecular and Surface Forces, Academic Press, London, 1991.

9 M. Bostrom, D. R. M. Williams and B. W. Ninham, Phys. Rev. Lett., 2001, 87, 168103.

10 N. Alcantar, J. N. Israelachvili and J. Boles, Geochim. Cosmochim. Acta, 2003, 67, 1289.

11 M. Dishon, O. Zohar and U. Sivan, Langmuir, 2009, 25, 2831.

12 G. V. Franks, J. Colloid Interface Sci., 2002, 249, 44.

13 S. Veeramasuneni, Y. Hu, M. R. Yalamanchili and J. D. Miller, J. Colloid Interface Sci., 1997, 188, 473.

14 E. Finot, E. Lesniewska, J.-C. Mutin and J.-P. Goudonnet, J. Chem. Phys., 1999, 111, 6590.

15 E. R. Beach, G. W. Tormoen, J. Drelich and R. Han, J. Colloid Interface Sci., 2002, 247, 84.

16 O. Zohar, I. Leizerson and U. Sivan, Phys. Rev. Lett., 2006, 96, 177802.

17 R. J. Flatt, J. Cryst. Growth, 2002, 242, 435-454. 